\title{
Validating a Questionnaire Assessing Knowledge and Awareness of Emergen- cy Trolleys: Revisions Required to Account for Variances in a Low Resource Setting
}

\author{
Charles Twagirayezu ${ }^{1}$, Stephanie Taylor ${ }^{1}$, Julie Pfeffer ${ }^{1}$ \\ ${ }^{1}$ University Teaching Hospital of Butare, Rwanda
}

\section{Background}

Nurses and doctors need knowledge about and access to resuscitation materials quickly and efficiently to improve the quality of emergency care. This is especially difficult in a low resource setting. To address this need, emergency trolleys are being developed on a unit-based level at a referral hospital in Rwanda. The purpose of this project is to identify the limitations encountered and overcame upon developing a questionnaire across multiple hospital units in a low resource setting.

\section{Description}

CHUB is a national referral level hospital in the Southern Province of Rwanda comprised of eleven clinical departments. A questionnaire was developed to assess the knowledge and awareness of nurses and doctors on the availability, knowledge, and use of emergency trolleys. In November 2015, the questionnaire was implemented. After 40 submissions across five departments, it was found that the questionnaire was not capturing the intended information. The initial questionnaire was not able to differentiate between partial understanding and no understanding of an emergency trolley or capture the difference in knowledge on necessary emergency trolley materials to consider it functional. Revisions were then made to the questionnaire to help account for these variables.

\section{Lessons Learned}

The initial questionnaire allowed those who had no knowledge of an emergency trolley to complete all items. Items on the questionnaire were poorly phrased, occasionally leading participants to answer incorrectly. For example, instead of asking yes or no to the question, Is the emergency trolley available in your service, the participant can now select from: yes, I have seen it; no, I have not seen it; or no, I do not know what an emergency trolley is.

\section{Conclusions}

Validating a questionnaire is critical to ensure the appropriate information is captured from the target audience. Creating questions in simple terms including all possible options is important. Questionnaires with specific answers help to fully identify the sought information, whether it be lack of available materials or knowledge. These findings can help formulate the proper formation of questionnaires to unveil the appropriate interventions to implement.

Key words: emergency trolley, tool validation, limited resource setting 